\title{
Cost-Sensitive Learning Based on Bregman Divergences ${ }^{\star}$
}

\author{
Raúl Santos-Rodríguez ${ }^{1}$, Alicia Guerrero-Curieses ${ }^{2}$, Rocío Alaiz-Rodríguez ${ }^{3}$, \\ and Jesús Cid-Sueiro ${ }^{1}$ \\ 1 Department of Signal Theory and Communications, \\ Universidad Carlos III de Madrid, Leganés (Madrid), Spain \\ \{rsrodriguez, jcid\}@tsc.uc3m.es \\ 2 Department of Signal Theory and Communications, Universidad Rey Juan Carlos, \\ Fuenlabrada (Madrid), Spain \\ alicia.guerrero@urjc.es \\ 3 Department of Electrical and Electronic Engineering, Universidad de León, León, \\ Spain \\ rocio.alaiz@unileon.es
}

\begin{abstract}
This paper analyzes the application of a particular class of Bregman divergences to design cost-sensitive classifiers for multiclass problems. We show that these divergence measures can be used to estimate posterior probabilities with maximal accuracy for the probability values that are close to the decision boundaries. Asymptotically, the proposed divergence measures provide classifiers minimizing the sum of decision costs in non-separable problems, and maximizing a margin in separable MAP problems.
\end{abstract}

Keywords: Cost sensitive learning, Bregman divergence, posterior class probabilities, maximum margin.

\section{Reference}

1. Santos-Rodríguez, R., Guerrero-Curieses, A., Alaiz-Rodríguez, R., Cid-Sueiro, J.: Cost-sensitive learning based on Bregman divergences. Machine Learning (2009) DOI: $10.1007 / \mathrm{s} 10994-009-5132-8$

\footnotetext{
* This is an extended abstract of an article published in the machine learning journal [1.
} 